\title{
ON THE MARKET
}

\section{SEEING RED}

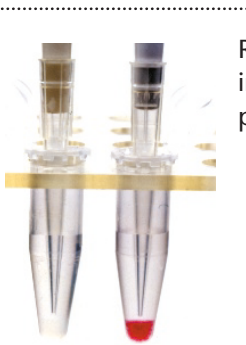

Red affinity gels for immunoprecipitation purposes.

Sigma-Aldrich offers two types of EZview red affinity gels, EZview Red Protein A affinity gel and EZview Red Anti-Flag M2 affinity gel, which provide enhanced visibility and performance equal to noncolored affinity gels used for immunoprecipitation. Most immunoprecipitation procedures have a number of washing steps, during which an affinity gel with specifically bound proteins undergoes centrifugation, and the resultant supernatant is aspirated from the affinity gel pellet. Non-colored affinity gel pellets are whitish or transluscent, making them very difficult to see in small polypropylene centrifuge tubes. Inadvertent aspiration of all or part of the gel pellet along with the supernatant can be a problem, resulting in loss of valuable data.

Tel. (+1) 314-771-5765

Fax (+1) 314-771-5757

www.sigma-aldrich.com

PCR ENZYMES

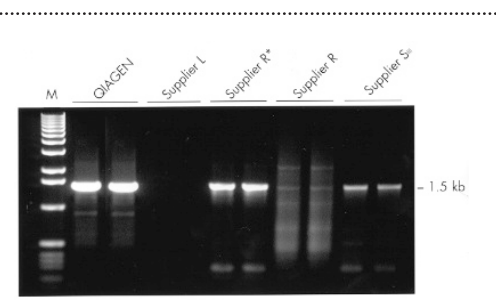

ProofStart DNA polymerase for high-fidelity PCR.

ProofStart DNA polymerase is the new proofreading enzyme from Qiagen designed for robust, high-fidelity PCR. The enzyme is chemically modified to inactivate both the polymerase and the exonuclease activity. Primers remain intact and mispriming cannot occur during setup, according to the company. A simple hot start allows PCR to start with high proofreading activity. A uniquely balanced combination of cations in the ProofStart PCR buffer provides stringent primer-annealing conditions over a wide range of conditions, says Qiagen. For templates with a high degree of secondary structure or a high GC content, ProofStart DNA polymerase is provided with Q-solution. This reagent modifies the melting behavior of DNA to improve amplification of such problematic templates.

Tel. (+49) (0) 2103-29-13384

Fax (+49) (0) 2103-29-23384

www.qiagen.com

SUBSTRATES

DuoLuX chemiluminscent/fluorescent substrate for alkaline phosphatase and peroxidase.

The DuoLuX chemiluminescent/fluorescent substrate from Vector Laboratories is now offered in two forms - one for development with alkaline phosphatase, the other for development with the peroxidase enzyme system. Both substrates can be used for western, Southern, northern or dot blots, or for ELISAs. They are said to offer high sensitivity, prolonged light emission and permanent fluorescence. For western blot chemiluminescent detection, the stated sensitivity is approximately $1 \mathrm{pg}$ of protein using either alkaline phosphatase or peroxidase. For nucleic acid detection applications, DuoLuX substrate for alkaline phosphatase is slightly more sensitive than DuoLuX substrate for peroxidase, enabling visualization of as low as $100 \mathrm{fg}$ of target nucleic acid, according to the company. DuoLuX substrate for peroxidase is a two-component system. The reagents when mixed in equal volumes yield $200 \mathrm{ml}$ of peroxidase working solution.

Tel. (+1) 650-697-3600

Fax (+1) 650-697-0339

www.vectorlabs.com

\section{PROBING MATTERS}

The InnoGenex Alu probe will detect Alu repeat sequences found in primate chromosomes in formalin-fixed, paraffin-embedded tissue sections by in situ hybridization. The probe is a mixture of synthetic oligonucleotides labeled with five fluorescein moieties at the $5^{\prime}$ end and is designed for efficient detection using anti-fluorescein antibodies. The probe should prove useful for the localization of human tissues in chimeric animals. Rodents with human cancer implants can be stained with the Alu probe to determine the extent of cancer proliferation.

Tel. (+1) 925-543-1400

Fax (+1) 925-543-1405

www.innogenex.com

\section{RECYCLING KITS}

Chemicon offers the ReBlot Plus western blot recycling kit. The kit contains two specially formulated antibody stripping solutions (mild and strong) that are designed to quickly and effectively remove antibodies and their corresponding chemiluminescent signal from membrane blots, without destroying the blotted protein sample. The mild stripping solution has been found to be sufficient for removing most antibodies, but the strong stripping solution may perform better when membranes with high signal are to be stripped or when ReBlot Plus mild treatment is not sufficient. The reagents are not recommended for stripping colorimetric substrates (TMB, DAB, 4chloronaphthol) and should be used for qualitative purposes until quantitative standards can be run in parallel.

Tel. (+1) 909-676-8080

Fax (+1) 909-676-9209

www.chemicon.com

\section{CELL ENRICHMENT}

Lineage-specific chimerism is often performed to monitor engraftment of minitransplants. StemCell Technologies now offers complete RosetteSep kits for enriching either myeloid or lymphoid cells specifically for this purpose. RosetteSep is designed as a rapid and easy procedure that enriches cells directly from whole blood with a simple centrifugation. The antibody-based RosetteSep enrichment cocktails contain reagents that specifically link red blood cells to unwanted cells in a sample of whole blood. When centrifuged over a specially designed density medium included in the kits, the desired cell population is highly enriched and easily recovered at the medium:plasma interface. The entire procedure is said to take an hour from start to finish.

Tel. (+1) 604-877-0713

Fax (+1) 604-877-0704

www.stemcell.com 